\title{
THE UNIVERSITY OF WARWICK
}

University of Warwick institutional repository: http://go.warwick.ac.uk/wrap

This paper is made available online in accordance with publisher policies. Please scroll down to view the document itself. Please refer to the repository record for this item and our policy information available from the repository home page for further information.

To see the final version of this paper please visit the publisher's website. Access to the published version may require a subscription.

Author(s): Green, R.J. Joshi, H. Higgins, M.D. Leeson, M.S.

Article Title: Recent developments in indoor optical wireless [Optical wireless communications]

Year of publication: 2008

Link to published article:

http://dx.doi.org/10.1049/iet-com:20060475

Publisher statement: "ㄷ 2008 IEEE. Personal use of this material is permitted. Permission from IEEE must be obtained for all other uses, in any current or future media, including reprinting/republishing this material for advertising or promotional purposes, creating new collective works, for resale or redistribution to servers or lists, or reuse of any copyrighted component of this work in other works."

Citation: Green, R. J. et al. (2008). Recent developments in indoor optical wireless [Optical wireless communications]. Communications, IET, Vol. 2 No. 1, pp. 3-10 


\title{
Recent Developments in Indoor Optical Wireless Systems
}

\author{
R. J. Green, H. Joshi, M. D. Higgins and M. S. Leeson \\ Roger.Green@warwick.ac.uk; H.S.Joshi@warwick.ac.uk; M.Higgins@warwick.ac.uk; \\ Mark.Leeson@warwick.ac.uk
}

All authors are with the:-

School of Engineering, University of Warwick, Coventry, CV4 7AL, UK.

First Submission Date: 15-08-2006

Revision Submission Date: 18-05-2007

\begin{abstract}
An overview of the developments in optical wireless systems viewed from the traditional communications viewpoint of transmitter, channel and receiver is presented. The trends in modulation formats that match information to the optical wireless channel are considered. This is followed by the discussion of recent transmitter and receiver innovations, particularly the utilisation of diversity transceivers. As a preliminary to the following treatment, the nature and modelling of the optical wireless channel are introduced, with particular emphasis on its unique features in terms of transmitted power constraints and non-negativity. From the examination of modulation formats, on-off-keying remains the format of choice for basic binary transmission, whereas
\end{abstract}


pulse-position modulation and its derivatives are preferred for more sophisticated requirements. The recent introduction of techniques from radio systems employing subcarriers is seen to be the most promising development in modulation techniques at present. In receiver technology, quasi-diffuse systems employing multispot diffusion and angular diversity are significant developments. They offer lower path loss and less multipath dispersion, at a lower transmission power compared to 'conventional' wide-angle diffuse systems, while providing a high level of user mobility compared to line-of-sight transmission. These developments are helping optical wireless systems to fulfil their promise by adopting a philosophy inspired by the radio domain to accommodate operation within a hostile channel.

\section{Introduction}

Optical wireless is an interesting technology combining the mobility of radio frequency (RF) wireless communications with the high bandwidth availability of optical communications. It offers the opportunity for high-speed communications and also has some of the properties of RF, such as the relative freedom of the user from fixed networks. The conventional RF community has now accepted that optical wireless is part of the electromagnetic spectrum, at least, in part, thanks to a Wireless World Research Forum White Paper [1].

The major design challenge, however, for such communication systems is in achieving a sufficiently high signal-to-noise ratio (SNR) at useful data rates. Since the SNR for an optical wireless link is proportional to the square of the average received optical signal power, transmission at higher power levels compared to the electrical channel is required. However, this power is limited by eye safety considerations [2], and portable device power consumption limitations imply that the system should be designed for minimum path loss. This requirement encourages 
suitable developments in receiver technology so as to be able to work with an acceptable level of path loss. Signal degradation also arises from the combined effects of ambient shot noise and intersymbol interference from multipath dispersion.

The optical wireless channel combines the filtered Gaussian-noise characteristics of conventional wire-based RF channels with the intensity modulation/direct detection (IM/DD) constraints of an optical fibre channel. The dominant source of noise in a non-directed optical wireless link is background illumination consisting of fluorescent light, sunlight and incandescent light [35].

In a diffuse link, the optical wireless channel, from transmitted signal power to the detected photocurrent, may be approximated by the additive white Gaussian-noise (AWGN) model as shown in Fig. 1a [6]. The high bit rates and relatively slow movement of transmitter and receiver mean that the channel impulse response varies appreciably only over distances comparable with the wavelength of the modulating signal. Hence, the optical wireless channel with diffused non-directed radiation is viewed as time invariant over short-time scales, as the channel response varies significantly only over a time period covering many symbols. The signal dispersion caused by multipath optical propagation is modelled as a linear baseband impulse response $r . h(t)$, where $r$ is the responsivity of the photodiode in Amperes per Watt.

A detailed channel model for a Line-of-Sight (LOS) link is presented in [7] and is depicted in Fig. 1b. The optical LOS link can be considered as non-frequency selective, with the detected radiant flux depending on the effective detector area, and the square of the distance between the transmitter and the receiver. Ambient radiation is modelled as AWGN with a high DC content. In addition to noiseinduced signal degradation, there is significant path loss because 
of the transmitterreceiver distance, and pulse extension that results from the larger time constant of the detected photocurrent's diffusion component. Much of the discussion in this paper is concentrated on indoor optical wireless communication systems, as it is more challenging to design an optical-LAN, and the incentives are enormous bandwidth coupled with high-speed communications.

Generally, the optical wireless channel is intensity modulated $[8,9]$, as the modulation of the phase or frequency of the light is more difficult. Since the signal modulates the optical intensity (instantaneous power), the transmitted waveform, $x(t)$, must be non-negative. The average transmitted power is the mean of the input power signal rather than the mean square of the signal amplitude, as in the case of the conventional linear Gaussian-noise channel. Also, as a result of eye safety considerations, the magnitude of the transmitted power must not exceed a prescribed value, say $P$.

Mathematically, the constraints on input signal in an optical wireless channel can be expressed as

$$
\begin{aligned}
& x(t) \geq 0 \\
& \lim _{T \rightarrow \infty} \frac{1}{2 T} \int_{-T}^{T} x(t) d t \leq P
\end{aligned}
$$

The other major feature is time dispersion arising from multipath effects in a diffuse link. Directed LOS links do not suffer from multipath dispersion [10], but require alignment between transmitter and receiver, limiting user mobility. This problem can be solved by using a quasidiffuse configuration, as discussed in Section 3, simultaneously maintaining multiple directed LOS links using space division multiple access. 


\section{Modulation}

The modulation procedure in IM/DD systems may entail two stages. The first is the optional modulation of a carrier frequency by the data signal, and the second is the modulation of the emitted infrared light. The low-frequency components close to DC should not be used for data transmission, to ensure stable and reliable signal transmission, because the receiver performance is dominated by shot noise generated by ambient illumination at low frequencies. Of course, alongside digital communication techniques there exist hybrid analogue RF-optical links, in which the particular advantages of optical wireless can be combined with RF [11], and these techniques may be extended for IR ad hoc networks [12].

\subsection{Modulation technique characteristics}

The quality of a modulation code is dictated by the reliability of transmission, the bandwidth efficiency and the power efficiency that it offers under typical noise and multipath conditions in the optical wireless channel.

\subsubsection{Reliability:}

A modulation technique should adapt the signal to the optical wireless channel in such a way that allows reception and consequent demodulation at the receiver end within the limit of an acceptable bit-error rate (BER). In particular, it should be able to deal with intersymbol interference and variations in the data signal DC content. Furthermore, in the case of short-range, mobile point-to-point links (where the electrical signal consists of high or low pulses) it is necessary that a long absence of a 'zero to one' transition does not prevent adequate sample clock recovery with a digital phase locked loop in the receiver [13]. In addition, the modulation technique should be resistant to modulation-independent effects of the channel such as phase jitter 
because of the variations of signal power, pulse extension because of the diffusion component's large time constant and pulse distortion from near-field signal clipping.

\subsubsection{Bandwidth Efficiency:}

Since the achievable bit-rate of an optical wireless communication system is limited by the bandwidth of the receiver electronics and multipath dispersion, it is important to use a modulation technique with high-bandwidth efficiency, $\eta_{B}$. This is defined as the ratio of the achievable bit-rate $\left(R_{\mathrm{bit}}\right)$ to the bandwidth $(B)$ of the transceiver, providing a measure of the utilisation of the bandwidth of the transceiver by a modulation technique, thus [7]

$$
\eta_{B}=\frac{R_{\mathrm{bit}}}{B}
$$

\subsubsection{Power Efficiency:}

The eye safety power limit suggests that the modulation techniques with a high peak-to-mean power ratio are favourable. The power efficiency $\left(\eta_{P}\right)$ of a modulation scheme is given by the average optical power required to achieve a given BER at a given data rate [6]. Mathematically, $\eta_{P}$ is defined as the ratio of energy per optical pulse to the mean energy required per bit, $E_{b}$, for a normalised transmit pulse shape, $p(t),[7]$ given by

$$
\eta_{P}=\frac{\int_{-\infty}^{\infty} p^{2}(t) d t}{E_{b}}
$$

When the modulation scheme does not utilise pulsed waveforms, for example, multiple subcarrier modulation, power efficiency is commonly measured in terms of normalised power requirement, defined as the ratio of the power required by the modulation scheme under consideration to the power required in the case of onoff keying (OOK), under the same conditions. 
The relationship between the power efficiency and bandwidth efficiency is [7]

$$
\eta_{P}=\frac{\eta_{B}}{\overline{\mathrm{DC}}}
$$

where $\overline{\mathrm{DC}}$ is the average duty cycle of the modulated signal, given by the average ratio of the high pulse duration to the low pulse duration during the transmission of a long data packet. These simple, essentially empirical quantities are commonly employed, since the problem of an optimal information-theoretic trade-off between bandwidth and power efficiencies for optical wireless channels remains unexplored.

\section{$2.2 \quad$ Specific modulation techniques}

\subsubsection{Binary modulation:}

A detailed comparative analysis of various binary modulation schemes including OOK, twopulse-position modulation (2-PPM) and subcarrier binary phase-shift keying (BPSK) is presented in [6]. It is shown that the power efficiency of OOK and 2-PPM is the same, whereas, since BPSK consists of half the transmit pulse energy compared to OOK, its power efficiency is $<3 \mathrm{~dB}$. Considering bandwidth efficiency, 2-PPM and BPSK have equal bandwidth requirements, being twice that needed for OOK. Thus, OOK emerges as the most suitable modulation scheme among binary modulation schemes, but at high data rates ( $>10 \mathrm{Mbps})$ it suffers from a large power penalty, because of multipath dispersion [14].

\subsubsection{Multilevel modulation:}

The possible candidates for multi-level modulation schemes in an intensitymodulated channel are: $L$-level baseband pulse-amplitude modulation ( $L$-PAM), subcarrier $L$-level quadratureamplitude modulation ( $L$-QAM), multiple-subcarrier modulation (MSM) and $L$-level PPM $(L$ PPM). 
In $L$-PAM, one of $L$ possible levels is transmitted during each symbol interval, resulting in a reduced symbol rate, and thus a reduced bandwidth requirement [6]. However, high-level PAM suffers from an increased power penalty compared to OOK, depending upon the value of $L$, arising from the increased size of symbol alphabet, with each symbol carrying $\log _{2} L$ bits of information.

Subcarrier $L$-QAM suffers from an additional power penalty of $1.5 \mathrm{~dB}$, because of the power wasted in the DC offset common to all subcarrier techniques, plus multisubcarrier and multilevel penalties [15]. Thus, compared with OOK, the power requirement to achieve a given bit rate is relatively higher for subcarrier L-QAM. For example, a single subcarrier 16-QAM system requires $4.8 \mathrm{~dB}$ more power compared with OOK for a given bit rate [6]. However, the bandwidth requirement is independent of the number of subcarriers, depending only on the alphabet size $L$.

In $L$-PPM, the symbol interval of duration $T$ is divided in to $L$ sub-intervals, and the optical signal is 'on' during lth sub-interval, and 'off' for the rest of the symbol duration. The $L$ possible signals form an orthogonal basis for the signal sub-space spanned by the transmitted signals [16]. The bandwidth of $L$-PPM is approximately the bandwidth of the 'on' pulse, and continues to grow with increasing $L$. However, for $L>2$, the power requirement of $L$-PPM is below that of OOK. In particular, 4-PPM has the same bandwidth efficiency as OOK, and requires $3.8 \mathrm{~dB}$ less optical power to achieve a given bit rate [6]. Table 1 summarises the power and bandwidth requirements of the modulation schemes considered so far.

As can be seen from Table 1, the power requirement for a multiple-subcarrier system is larger 
than that for a single subcarrier system, and therefore the resistance to multipath dispersion provided by using multiple-subcarriers is offset by the increased power requirement. Also, subcarrier techniques suffer from the increased attenuation of the multipath frequency response at high frequencies when compared with baseband systems, so the intensity-modulation channel favours baseband modulation schemes with low duty cycles.

Nevertheless, the resilience of MSM systems in the presence of multipath dispersion makes them attractive for indoor optical wireless communications. Thus, much recent effort has been directed towards improving the power efficiency of MSM systems. You and Kahn [17] proposed two simple and effective approaches. The first employs block coding between the information bits and the subcarrier modulating symbol amplitudes to increase the minimum value of the multiple-subcarrier electrical waveform. The second replaces the fixed DC bias by a signal that varies on a symbol-by-symbol basis. Using eight BPSK or QPSK subcarriers, these techniques give average power reductions of $\sim 3.6$ and $3.2 \mathrm{~dB}$, respectively [17].

Another method to improve the power efficiency in MSM systems is described in [18], where a suboptimum set of $L$, the peak reduction carriers (PRC), from $N$ subcarriers, is transmitted, with the aim of reducing the DC bias. This MSM-PRC system selects the optimum set of phases and amplitudes, so that the average optical power requirement is reduced, mimicking what is done in orthogonal-frequency division multiplexed (OFDM) signals [19]. The information bits are mapped onto each subcarrier other than the PRCs independently, and hence the error rate of the proposed system is unaffected by the PRCs. It is shown in [18] that the normalised power requirement for a proposed MSM system with a single peak reduction carrier is $0.18 \mathrm{~dB}$ smaller than for a conventional MSM system, at a normalised bandwidth requirement of 1.5. 


\subsection{Recent advances in modulation techniques}

\subsubsection{Differential PPM (DPPM):}

The DPPM is a simple modification of PPM that can achieve improved power and/ or bandwidth efficiency, and is superior to PPM in optical multiple-access applications. A DPPM symbol is obtained from the corresponding PPM symbol by deleting all of the 'off' chips following the 'on' chip. DPPM detection does not require symbol synchronisation, which is necessary in case of PPM detection. It is shown in [20] that, for a given bandwidth, the DPPM requires significantly less power than PPM. For example, the 16-DPPM provides a $3 \mathrm{~dB}$ optical average power gain over 4-PPM and requires only slightly more bandwidth. The 16-DPPM also supports the same bit rate as 16-PPM using less than half of the bandwidth, but at $1.4 \mathrm{~dB}$ higher average power. The reason lies in the fact that in DPPM, the redundant 'off' chips are omitted without significantly affecting the error probability. Therefore for a fixed average bit rate and fixed bandwidth, a higher $L$ can be employed with DPPM compared to PPM, resulting in improved power efficiency. However, an unusual characteristic of DPPM is the variable bit rate nature. This is because of the fact that DPPM symbols do not have equal durations and therefore the time required to transmit a packet containing a fixed number of bits is not constant [18]. This may result in transmitter buffer overflow/ underflow from packets requiring excessive/too little transmission time, temporary violation of eye safety laws if the packet transmission time is too small, and inefficient utilisation of network resources from the provisioning for delayed data packets. Several techniques to reduce the bit rate variations were proposed in [20].

\subsubsection{Trellis-coded PPM:}

Trellis-coded modulation is designed to maximise the minimum Euclidean distance between allowed signal sequences by using signal-set expansion to provide redundancy for coding, and 
by using set partitioning in code design. Trellis-coded PPM has been shown to be very effective in combating optical wireless channel multipath dispersion [21]. Multipath dispersion causes symbols, whose chips are adjacent in time, to be separated by smaller Euclidean distances than symbols whose chips are well separated in time. Hence, as the multipath-induced intersymbol interference increases, the effectiveness of a Trellis-coded modulation scheme also increases. The results obtained in [21] indicate that rate-2/3-coded 8-PPM has electrical gains of up to $5 \mathrm{~dB}$ over uncoded 16-PPM for a constraint-length-10 code, with the same bandwidth requirement. However, 7 is a more practical value of constraint length, used in the IEEE 802.11 standard to reduce the complexity of the Viterbi decoder.

\subsubsection{Rate-adaptive modulation techniques:}

Although the optical wireless channel can support high bit rate transmission, it is not possible to maintain constantly the SNR to support this because of abrupt changes in ambient noise and variations in transmitterreceiver distance. Under adverse conditions, it is important to maintain the link by reducing the data rate until an acceptable BER is achieved. Rate-adaptive transmission is proposed in [22] using simple repetition codes and rate-compatible punctured convolutional codes combined with $L$-PPM. Repetition codes consist of repetition of each $L$-PPM symbol RR times, where $R R$ is the rate reduction factor. The receiver estimates the channel conditions, and thus the data rate that can be supported for a given BER. This recommended bit rate is conveyed to the transmitter via a feedback channel for adjustment of the transmission rate.

Another rate-adaptive modulation scheme is based on the use of variable silence periods and OOK formats with memory [23]. The former are used to modify the statistics of the amplitude sequence related to the message sequence, providing an increase in the peak-to-average optical 
power ratio. This scheme provides a signalling rate with wide dynamic range, and results in reduced complexity compared with the scheme based on repetition coding specified in the advanced infrared (AIr) standard [24].

Both techniques mentioned above represent approaches in the time domain but recently the frequency domain-based method of adaptively multiplexing orthogonal subcarriers has emerged [25]. This technique is analogous to adaptive OFDM in the RF domain. The proposed system dynamically adjusts the transmission rate to the optical wireless channel conditions by assigning suitable modulation formats to individual subcarriers to fully utilise the channel capacity. The rate-adaptation is maintained even under a worst-case scenario by using the lowest subcarriers (which are always suitable for transmission in the case of an optical wireless channel) over a low-rate feedback link. The data rate is chosen on a carrier-by-carrier basis, and therefore the system is able to adapt to a wide range of channel conditions. The throughput benefits of the HughesHartog bit-loading algorithm over straightforward bit allocation, based on optimal power distribution, are demonstrated in [25]. This work demonstrates that the high-level modulation formats (QPSK, 16-QAM, 64-QAM) are efficient with benign channel conditions, whereas lowcomplexity modulation formats, such as PPM, are necessary to maintain reliable transmission under adverse channel conditions.

In [26], a capacity analysis is performed for a system using adaptive multiple-subcarrier transmission. An algorithm is developed using Lagrange multipliers for optimal optical power allocation to individual subcarriers (which is analogous to the 'water-filling problem' in radio channels), and results are derived for the theoretically achievable data-rates under different channel conditions. An MSM optical wireless system, with $N$ orthogonal subcarriers, is considered as $N$ 
parallel-independent Gaussian channels with independent noise, and the channel capacity is given as the sum of capacities of the individual carriers. This work demonstrates the attractiveness of adaptive modulation, by exploiting the wide-varying optical wireless communication channel characteristics.

\subsubsection{Edge position modulation (EPM):}

The EPM technique divides the transmission time interval into discrete slots, each of which is slightly greater than the rise time interval plus the jitter interval of the rising edge of the transmitted pulses [7]. Since the rise time and jitter interval are much smaller than the pulse length, the time slots for EPM can be smaller than the time slots for PPM. Thus, with EPM, more information can be transmitted within a certain transmission interval, as there are more possibilities to arrange the pulses compared to PPM. The coding used in the EPM scheme is run-length-limited (RLL) coding, ensuring that the rising edge of the pulse is not disturbed by the previous pulse, and thus avoiding intersymbol interference. The code input is the bit stream to be transmitted, and the outputs specify the positions where the pulses should start. The RLL coding allows EPM to operate with relatively large pulse extensions, and hence is well adapted to the optical wireless channel, offering increased bandwidth efficiency at reasonable power efficiency [7].

\subsubsection{Digital pulse interval modulation (DPIM):}

In DPIM, each block of $\log _{2} L$ data bits is mapped onto one of $L$ possible symbols, each differs in length [27]. Information is encoded by varying the number of empty slots between adjacent pulses, and hence the redundancy of empty slots following a pulse in PPM symbol is removed. Each symbol is initiated with a pulse and is followed by a number of empty slots directly dependent on the decimal value of the block of data bits being encoded. The variable symbol duration 
means that the overall bit rate is variable in DPIM, and the slot rate is selected such that the mean symbol duration is equal to the time taken to transmit the same number of bits using OOK or $L-P P M$ achieving the same average bit rate.

The unique symbol structure of DPIM is exploited in combating intersymbol interference by introducing guard slots in each symbol immediately following the pulse. During detection, the slots contained within the guard slot following the correctly detected pulse are automatically assigned zero values, regardless of the level of the sampled output. The error performance with/without equalisation for DPIM is presented in [27].

\section{Transmitter and receiver aspects}

As with any mobile communication system, the objective is to provide the user with a high degree of movement, coupled with a high data rate. In addition to modulation aspects, the transmitter and receiver designs are also important factors in system performance. The design techniques employed to realise them should take into account the configuration of the system.

A diffuse system requires a high-power transmitter and a sensitive receiver, because of the high path loss from the multiple bounces incurred between the source and the receiver, limiting the rate of modulation that can be achieved [28]. The low level of optical power often present at the receiver makes the utilisation of optical concentrators to maximise light collection beneficial [29]. However, the high level of ambient light presents results in shot noise from the entire fieldof-view (FOV) of the optical concentrator plus detector combination. This must be ameliorated by optical and electrical filtering [30]. The LOS channel offers higher speed because of the lower path loss, but requires a high degree of alignment, which cannot be blocked, limiting user 
mobility. Photogenerated noise can also be reduced because of the narrower FOV employed in the optical front-end of the receiver. An approach described in the recent literature is the quasi-diffuse, or tracked system, employing a multi-beam transmitter and an angle diversity receiver.

\subsection{Quasi-diffuse transmitters}

The purpose of the quasi-diffuse transmitter is to create multiple narrow beams targeted in different directions. By using such beams, the system requires lower transmitter power, has a lower path loss and reduced multipath dispersion, in comparison with single wide-angle transmitters, but the user still has freedom from link blockage. Fig. 2 shows some of the possible multispot diffusion patterns that can be achieved using quasi-diffuse transmitters, including a novel line strip multi-spot system [31].

Multispot diffusion can be achieved by implementing arrays of illuminating elements, coupled to conventional lenses or computer-generated holograms [32]. It is also possible to target the narrow fields towards a reflective surface, such as the ceiling as shown in Fig. 2, or provide many direct LOS links from a base station situated on the ceiling, projecting down onto the receiver $[33]$.

\subsection{Quasi-diffuse receivers}

Angular diversity receivers (ADRs) utilise an array of narrow FOV receiving elements that are pointed in different directions and/or have different spatial locations, combining to create a wide-angle receiver. Each element has its own amplifier circuitry, and the system may employ signal processing to determine which elements are receiving signals, allowing the rejection of some inputs or power saving by deactivating those elements receiving only noise. Multipath 
dispersion effects can also be mitigated by rejecting elements that receive weak signals from reflections if an LOS link is present. Another advantage is that each element with a narrow FOV is smaller than a single element with a wide FOV, so the capacitance of each detector is smaller, and thus increasing the bandwidth of the system. The disadvantage of using ADRs is the added complexity of the receiver, which has to incorporate multiple detectors, each with its own amplifier and an overall processor.

The ADR can be either imaging or non-imaging, as shown in Fig. 3. The former employs only one imaging optical concentrator that forms an image of the received light onto the photodetector array, thus sending the light that arrives from different directions to different detectors. The latter couples each element to a non-imaging optical concentrator with a filter, and the receiver can then determine from where the light arrived via knowledge of the receiver orientation and the FOV attained by each element/concentrator.

A number of non-imaging concentrators have been proposed in the past, two of the most-popular being the hemispherical (truncated spherical) dielectric concentrator [3437], and the rotationally symmetric compound parabolic concentrator (CPC) [38, 39]. Hemispherical concentrators achieve a high FOV and gain, provided the hemisphere radius is large compared with the detector [30], and an optical filter can be placed between the concentrator and the detector or on the surface of the hemisphere. Although the filter placed on the surface of the hemisphere provides a $3 \mathrm{~dB}$ power advantage, it has to be traded off with the increased complexity of filter fabrication [10]. CPCs on the other hand can be designed for any FOV, and utilise thin film planar optical filters that are easier to fabricate and tune to a desired central wavelength in the bandpass region. Owing to the fact the FOV can be controlled, a higher optical gain can be achieved along 
with rejecting more background illumination. Recently, another alternative has been presented in the form of a three dimensional dielectric totally internally reflecting concentrator or optical antenna which also uses a planar optical filter between the concentrator and the detector, but, unlike the CPC, it can have a variety of entrance aperture shapes, giving it a smaller size and the ability for higher optical gain compared to the traditional CPC [29, 40].

Imaging ADRs offer two advantages. First, the bulk size of the receiver is reduced, as only one imaging optical concentrator is needed for the whole device. Second, the photodiode array is flat and may be fabricated into a highly scalable integrated circuit, offering high pixel density [41].

As stated above, the ADR requires signal processing to determine which pixels should be used. In [42], a system using multiple narrow beam transmitters and multiple narrow FOV receivers, transmitting at 70 Mbps over a range of $4.2 \mathrm{~m}$ using OOK with a BER of $10^{-9}$, compared the effectiveness of different combining schemes. Maximal ratio combining (MRC), which performs a weighted sum based on the signal amplitude to noise power spectral densities, and low-pass filtering with a cut-off point appropriate to the modulation speed, emerged as the most effective signal-combining scheme.

To quantify the effectiveness of using a quasi-diffuse system, Kahn et al. [41] compared an imaging ADR system transmitting at $30 \mathrm{Mbps}$ using OOK modulation targeting a BER of $10^{-9}$ with a conventional wide FOV single element receiver. It was found that, when considering LOS links from a wide-angle transmitter, the use of a 7 pixel imaging receiver required $3.7 \mathrm{~dB}$ less power than a singleelement receiver with equivalent optical properties. Furthermore, if the 
number of pixels was increased to over 1000, then a $13 \mathrm{~dB}$ transmitter power reduction was achieved. The work also compared MRC with simply selecting the pixel with the highest SNR, and demonstrated that MRC required slightly less power. Moreover, if the wide angle LOS transmitter was replaced by a multi-spot transmitter, creating a diffuse signal on the ceiling this particular configuration required $17 \mathrm{~dB}$ less transmitted power. Employing the multi-pixel ADR arrangement led to gains of up to $12 \mathrm{~dB}$ for greater than 1000 pixels.

In [43], the development of a combined directed (for high-speed) and diffuse link (for connectivity) system was demonstrated. In this approach, the diffuse link was provided by a high-power laser directed at a diffusely radiating surface, and the directed link was implemented with a vertical cavity surface emitting laser diode array with focusing lens. The imaging ADR in this work utilised an array of avalanche photodiodes to take the advantage of their higher sensitivity because ambient-related shot noise has less effect on diversity receivers. The use of a microcontroller in the ADR allowed all the pixels to be active if no LOS link was present, or selectively activated individual pixels for LOS transmission. To obtain error-free data transmission between the transmitter and the receiver at $1.8 \mathrm{~m}, 400 \mathrm{~mW}$ of power was required by the diffuse laser transmitter, whereas only $0.35 \mathrm{~mW}$ was required by the LOS-directed laser diode transmitter. The final system capability demonstrated was 155 Mbps over a range of $2 \mathrm{~m}$, using a $233 \mathrm{MHz}$ DBPSK subcarrier.

The first fully integrated quasi-diffuse system was presented in [44], using custom electronics to obtain improved performance over commercially available components. The transmitter consisted of an array of resonant cavity LED's flip-chip bonded to CMOS driver circuitry and integrated with the transmitter optics. A wavelength of $980 \mathrm{~nm}$ was employed because current 
wavelength devices are not advanced enough for the level of integration intended and this restricted transmitter power for eye safety reasons. The imaging ADR consisted of an array of pin photodiodes flip-chip bonded to custom CMOS circuitry. The use of integrated circuitry offers good scalability, and employing CMOS permits custom fabrication of low-cost, highly functional devices [33]. The aim of the project was to reach $155 \mathrm{Mbps}$ over $23 \mathrm{~m}$, and, to date, individual component testing within the link indicates that this will soon be accomplished.

Finally, a novel form of imaging receiver was presented in [45]. This consisted of a single photodetector, placed on a miniaturised positioning mechanism that allowed it to move under the focal plane of the imaging lens. Advantage was taken of the slow variation in the signal position occurring in mobile systems, which permitted searching for the best SNR via a simple search algorithm in real time. The system has similar benefits to the others described, in that ambient light is rejected and power can be saved at the transmitter by reducing path loss through LOS transmission. One drawback shown in the paper was that when the signal link was completely blocked, the system had to search again for a connection. The aim of this project, albeit in the simulation stage, was aimed at portable equipment applications, as the receiver complexity was low, yet still it was able to maintain the advantages of diversity receivers over those using single detector, wideangle receivers. The initial published simulation results predicted a $50 \%$ power saving at the transmitter by using the single-channel imaging receiver, compared to a similar angle diversity receiver, while also increasing the available channel bandwidth.

\section{Conclusions}

Optical wireless systems have now been with us for $>30$ years, but are seen by many as still an area of unfulfilled promise. In this work, we have examined recent new approaches to the diffi- 
culties of employing optical wireless to unlock the promise of unregulated bandwidth, security and freedom from EMC problems. After briefly introducing the optical wireless channel, this paper addressed the optical wireless system from the traditional communications viewpoint of transmitterchannelreceiver.

When using binary modulation, OOK is preferred for simplicity, even though it suffers somewhat from multipath dispersion at high bit rates. For more sophisticated requirements, PPM is attractive, with 4-PPM offering the BER performance of OOK but needing only $40 \%$ of the power. Adding trellis coding to PPM further increases its advantage for the price of a more complex receiver. Nevertheless, PPM is not straightforward to synchronise, and this has led to the development of DPPM, which operates at a variable bit rate. There is also the truly asynchronous scheme of DPIM that ameliorates the effects of intersymbol interference. In a different vein, EPM using RLL coding to avoid intersymbol interference has also emerged over the last few years. The most radical innovations in modulation schemes, however, have come with the employment of rateadaptive methods inspired by the RF domain. In the time domain, coding and silence periods have been employed, whil in the frequency domain OFDM has found application. It is in this final area that the most promising developments are occurring, and these will enable optical wireless communication systems to move forward from the days of low data rate and custom laboratory demonstrations.

In the receiver domain, the introduction of ADRs has produced substantial performance gains. Although ADRs can be imaging or non-imaging, the former offers the greatest advantages, permitting a relatively small receiver and straightforward system integration. Employing ADRs provides gains of $>10 \mathrm{~dB}$, when arrays of 1000 or more pixels are fabricated and controlled by 
integrated circuitry.

The adoption of RF-like methods to combat the unfriendly aspects of the optical wireless channel offers the prospect of considerable future success. In particular, this takes optical wireless closer to commercial operation as a viable alternative to the software-only security used in standard lower frequency RF systems.

\section{References}

[1] O’Brien, D.C., Katz, M., Wang, P., Kalliojarvi, K., Arnon, S., Matsumoto, M., Green, R.J., and Jivkova, S.: 'Short-range optical wireless communications' (Wireless World Research Forum, 2005)

[2] BS EN 60825-1:1994: 'Safety of laser products, equipment classification, requirements and user's guide', 1994

[3] Boucouvalas, A.C.: 'Indoor ambient light noise and its effect on wireless optical links', IEE Proc., Optoelectron., 1996, 143, pp. 334-338

[4] Moreira, A.J.C., Valadas, R.T.O., and Duarte, A.M.: 'Performance of infrared transmission systems under ambient light interference', IEE Proc., Optoelectron., 1996, 143, pp. 339-346 [5] Narasimhan, R., Audeh, M.D., and Kahn, J.M.: 'Effect of electronic-ballast fluorescent lighting on wireless infrared links', IEE Proc., Optoelectron., 1996, 143, pp. 347-354

[6] Barry, J.R.: 'Wireless infrared communications' (Kluwer Academic Press, 1994)

[7] Lueftner, T., Kroepl, C., Huemer, M., Hausner, J., Hagelauer, R., and Weigel, R.: 'Edge-position modulation for high-speed wireless infrared communications', IEE Proc., Optoelectron., 2003, 150, pp. $427-437$

[8] Korevaar, E.J.: 'Optical Wireless Communications IV', Proc. SPIE., 2001, 4530 
[9] Korevaar, E.J.: 'Optical Wireless Communications V', Proc. SPIE., 2002, 4873

[10] Kahn, J.M., and Barry, J.R.: 'Wireless infrared communications', Proc. IEEE, 1997, 85, pp. 265-298

[11] Green, R.J., Sweet, C., and Idrus, S.M.: 'Optical wireless links with enhanced linearity and selectivity [Invited]', J. Opt. Netw., 2005, 4, pp. 671-684

[12] Abu Baker, Z.: 'Infrared ad-hoc networking', PhD thesis, University of Warwick, 2007

[13] Hirt,W., Hassner,M., and Heise, N.: 'IrDA-VFIr (16 Mb/s):modulation code and system design', IEEE Pers. Commun., 2001, 8, pp. 58-71

[14] Wong, K.K., O'Farrell, T., and Kiatweerasakul, M.: 'The performance of optical wireless OOK, 2-PPM and spread spectrum under the effects of multipath dispersion and artificial light interference', Int. J. Commun. Syst., 2000, 13, pp. 551-576

[15] Carruthers, J.B., and Kahn, J.M.: 'Multiple-subcarrier modulation for nondirected wireless infrared communication', IEEE J. Sel. Areas in Commun., 1996, 14, pp. 538-546

[16] Audeh, M.D., Kahn, J.M., and Barry, J.R.: 'Performance of pulse-position modulation on measured non-directed indoor infrared channels', IEEE Trans. Commun., 1996, 44, pp. 654-659 [17] You, R., and Kahn, J.M.: 'Averagepower reduction techniques for multiple-subcarrier optical intensity modulation', IEE Colloquium 99/ 128, 'Optical Wireless Communications', 22 June 1999, paper 6

[18] Teramoto, S., and Ohtsuki, T.: 'Multiple-subcarrier optical communication system with peak reduction carriers', Trans. IEICE, 2004, E87-B, pp. 3385-3388

[19] Hanzo, L., Munster, M., Choi, B.J., and Keller, T.: 'OFDM and MC-CDMA for Broadband Multi-User Communications, WLANs, and Broadcasting' (Wiley Press, 2003)

[20] Shiu, D.S., and Kahn, J.M.: 'Differential pulse-position modulation for power-efficient optical communication', IEEE Trans. Commun., 1999, 47, pp. 1201-1210 
[21] Lee, D.C.M., Kahn, J.M., and Audeh, M.D.: 'Trellis-coded pulse-position modulation for indoor wireless infrared communications', IEEE Trans. Commun., 1997, 45, pp. 1080-1087 [22] Diana, L., and Kahn, J.M.: 'Rate-adaptive modulation techniques for infrared wireless communications'. IEEE Int. Conf. Commun. (ICC 1999), pp. 597-603

[23] Garcia-Zambrana, A., and Puerta-Notario, A.: 'Novel approach for increasing the peak-to-average optical power ratio in rate-adaptive optical wireless communication systems', IEE Proc., Optoelectron., 2003, 150, pp. 439-444

[24] IrDA: Advanced infrared physical layer specification, (AIrPHY)-Version 1.0, 1998

[25] Grubor, J., Jungnickel, V., and Langer, K.: 'Rate-adaptive multiple sub-carrier-based transmission for broadband infrared wireless communication'. National Fiber Optic Engineers Conf., 2006, p. 10

[26] Grubor, J., Jungnickel, V., and Langer, K.: 'Capacity analysis in indoor wireless infrared communication using adaptive multiple subcarrier transmission'. Proc. 7th Int. Conf. Transparent Optical Networks 2005 (ICTON 05), pp. 171-174

[27] Ghassemlooy, Z., Hayes, A.R., and Wilson, B.: 'Reducing the effects of intersymbol interference in diffuse DPIM optical wireless communications', IEE Proc., Optoelectron., 2003, 150, pp. $445-452$

[28] Carruthers, J.B., and Kahn, J.M.: 'Modeling of nondirected wireless infrared channels', IEEE Trans. Commun., 1997, 45, pp. 1260-1268

[29] Ramirez-Iniguez, R., and Green, R.J.: 'Optical antenna design for indoor optical wireless communication systems', Int. J. Commun. Syst., 2005, 18, pp. 229-245

[30] Street, A.M., Stavrinou, P.N., O'Brien, D.C., and Edwards, D.J.: 'Indoor optical wireless systems a review', Opt. Quant. Electron., 1997, 29, pp. 349-378

[31] Al-Ghamdi, A.G., and Elmirghani, J.M.H.: 'Spot diffusing technique and angle diversity 
performance for high speed indoor diffuse infra-red wireless transmission', IEE Proc., Optoelectron., 2004, 151, pp. 46-52

[32] Eardley, P.L., Wisely, D.R., Wood, D., and McKee, P.: 'Holograms for optical wireless LANs', IEE Proc., Optoelectron., 1996, 143, pp. 365-369

[33] O’Brien, D.C., Faulkner, G.E., Jim, K., Zyambo, E.B., Edwards, D.J., Whitehead, M., Stavrinou, P., Parry, G., Bellon, J., Sibley, M.J., Lalithambika, V.A., Joyner, V.M., Samsudin, R.J., Holburn, D.M., and Mears, R.J.: 'High-speed integrated transceivers for optical wireless', IEEE Commun. Mag., 2003, 41, pp. 58-62

[34] Savicki, J.P., and Morgan, S.P.: 'Hemispherical concentrators and spectral filters for planar sensors in diffuse radiation fields', Appl. Opt., 1994, 33, pp. 8057-8061

[35] Marhic, M.E., Kotzin, M.D., and van den Heuvel, A.P.: 'Reflectors and immersion lenses for detectors of diffuse radiation', J. Opt. Soc. Am., 1982, 72, pp. 352-355

[36] Kahn, J.M., Barry, J.R., Audeh, M.D., Carruthers, J.B., Krause, W.J., and Marsh, G.W.: 'Non-directed infrared links for high-capacity wireless LANs', IEEE Pers. Commun., 1994, 1, p. 12

[37] Barry, J.R., and Kahn, J.M.: 'Link design for non directed wireless infrared communications', Appl. Opt., 1995, 34, pp. 3764-3776

[38] Ho, K., and Kahn, J.M.: 'Compound parabolic concentrators for narrowband wireless infrared receivers', Opt. Eng., 1995, 34, pp. 1385-1395

[39] Wasiczko, L.M., Smolyaninov, I.I., and Davis, C.C.: 'Analysis of compound parabolic concentrators and aperture averaging to mitigate fading on free-space optical links', Free Space Laser Communication and Active Laser Illumination III (Eds. Voelz, D. G. and Ricklin, J. C.), Proc. SPIE, 2004, 5160, pp. 133-142

[40] Green, R.J.: 'Polarising concentrator for IR wireless'. GB0308128.8, July 2003 
[41] Kahn, J.M., You, R., Djahani, P., Weisbin, A.G., Teik, B.K., and Tang, A.: 'Imaging diversity receivers for high-speed infrared wireless communication', IEEE Commun. Mag., 1998, 36, pp. $88-94$

[42] Carruthers, J.B., and Kahn, J.M.: 'Angle diversity for nondirected wireless infrared communication', IEEE Trans. Commun., 2000, 48, pp. 960-969

[43] Jungnickel, V., Forck, A., Haustein, T., Kruger, U., Pohl, V., and von Helmolt, C.: 'Electronic tracking for wireless infrared communications', IEEE Trans. Wirel. Commun., 2003, 2, (5), pp. 989-999

[44] O’Brien, D.C., Faulkner, G.E., Zyambo, E.B., Jim, K., Edwards, D.J., Stavrinou, P., Parry, G., Bellon, J., Sibley, M.J., Lalithambika, V.A., Joyner, V.M., Samsudin, R.J., Holburn, D.M., and Mears, R.J.: 'Integrated transceivers for optical wireless communications', IEEE J. Sel. Top. Quantum Electron., 2005, 11, pp. 173-183

[45] Castillo-Vazquez, M., and Puerta-Notario, A.: 'Single-channel imaging receiver for optical wireless communications', IEEE Commun. Lett., 2005, 9, pp. 897-899 
Table 1: Comparison of various binary and multilevel modulation schemes, adapted from [6]

\begin{tabular}{lll}
\hline Modulation Scheme & Optical power requirement & Bandwidth requirement \\
\hline OOK & $\sqrt{ } N_{0} R_{B} Q^{-1}(\mathrm{BER})$ & $R_{B}$ \\
2 -PPM & $\sqrt{ } N_{0} R_{B} Q^{-1}(\mathrm{BER})$ & $2 R_{B}$ \\
BPSK & $\sqrt{ } 2 N_{0} R_{B} Q^{-1}(\mathrm{BER})$ & $2 R_{B}$ \\
$L$-PPM & $\left(L-1 / \sqrt{ } \log _{2} L\right) \sqrt{ } N_{0} R_{B} Q^{-1}(\mathrm{BER})$ & $R_{B} / \log _{2} L$ \\
$\mathrm{~N}$ - $L$-QAM & $\sqrt{ } 4 N\left(\sqrt{ } L-1 / \sqrt{ } \log _{2} L\right) \sqrt{ } N_{0} R_{B} Q^{-1}(\mathrm{BER})$ & $2 R_{B} / \log _{2} L$ \\
$L$-PPM & $\left(1 / \sqrt{ } 0.5 L \log _{2} L\right) \sqrt{ } N_{0} R_{B} Q^{-1}(B E R)$ & $L R_{B} / \log _{2} L$ \\
\hline
\end{tabular}

$N_{0}$, noise power spectral density

$R_{B}$, required bit rate

(a)
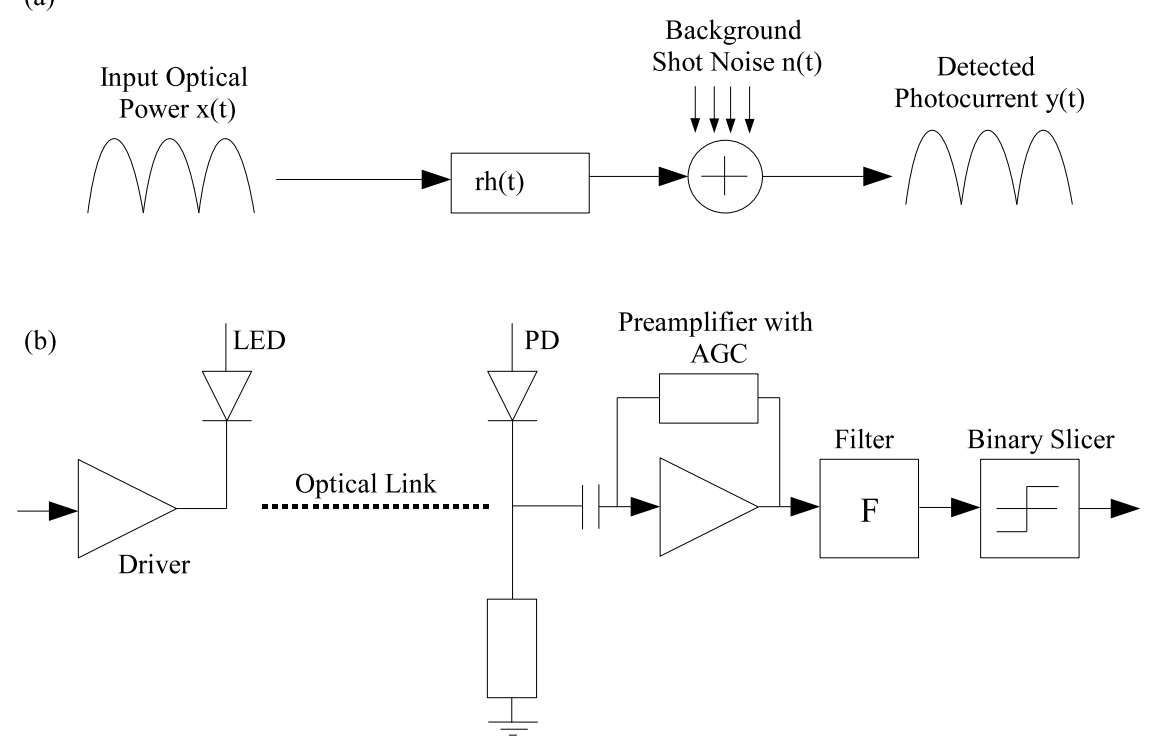

Figure 1: Channel modes for optical wireless transmission. (a) Equivalent channel model for diffused non-directed optical wireless link. (b) Physical model of line-of-sight (LOS) optical wireless link. 


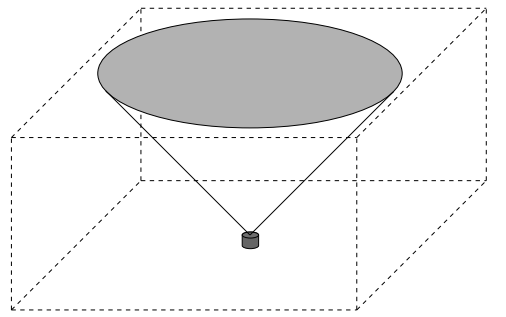

(a). Conventional Diffuse Transmitter

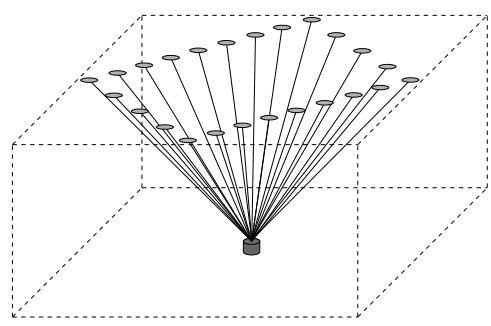

(c). Diamond Multispot Diffusion

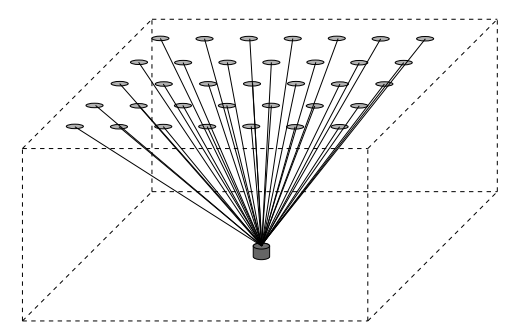

(b). Uniform Multispot Diffusion

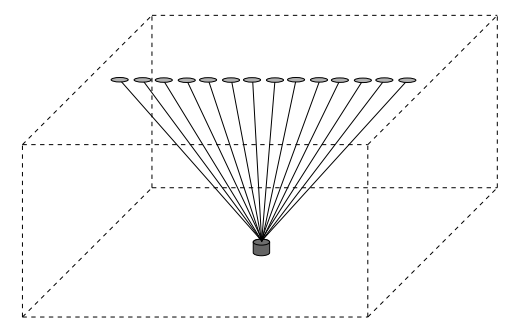

(d). LSMS Diffusion

Figure 2: Types of multispot diffusion patterns.

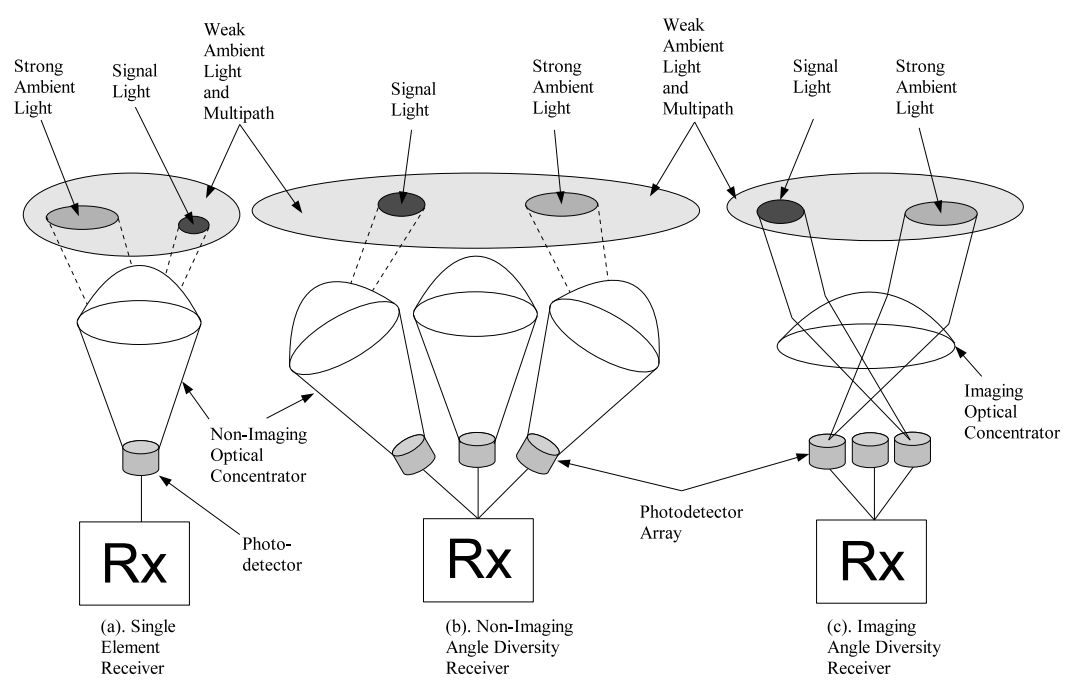

Figure 3: Receiver configurations. (a) Single element receiver. (b) Angle diversity receiver. (c) Imaging angle diversity receiver. 